\title{
Avaliação do efeito da obesidade infantil e a do adolescente sobre as propriedades ventilométricas e força muscular do sistema respiratório
}

\author{
Evaluation of the effect of childhood and adolescent obesity on the \\ ventilometric properties and muscle strength of the respiratory system
}

\author{
Viviani da Silva Soares Teixeira ${ }^{1}$, Brenda Camola Anjos Fonsecal, Daniel Martins Pereira², \\ Baldomero Antonio Kato da Silva ${ }^{3}$, Filipe Abdalla dos Reis ${ }^{4}$

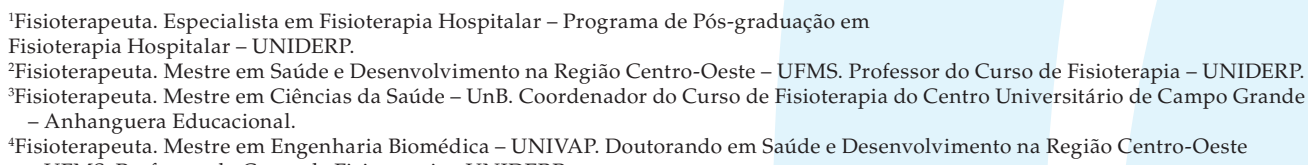

\section{Resumo}

Neste estudo, avaliaram-se as alterações na função pulmonar de 50 indivíduos (crianças e adolescentes obesas), pertencentes a dois grupos: sobrepeso e controle, submetidos ao teste de função pulmonar por meio da análise dos seguintes parâmetros: capacidade vital lenta (CVL), pico de fluxo expiratório (PFE) e força muscular respiratória pela medida das pressões respiratórias máximas (Pimáx e Pemáx). A CVL, para o grupo sobrepeso, foi, em média, de 3,0 $\pm 0,7$ litros, e para o grupo controle, de $3,49 \pm 0,9$ litros $(\mathrm{p}=0,0490)$. O PFE para o grupo sobrepeso registrou $336 \pm 107,4 \mathrm{~L} / \mathrm{min}$, e para o grupo controle, $386 \pm 55,5 \mathrm{~L} / \mathrm{min}(\mathrm{p}=0,0404)$. Concluiuse que houve alteração estatisticamente significativa na função pulmonar para as crianças e adolescentes obesos em relação a capacidade vital lenta e ao pico de fluxo expiratório. Não foi observada diferença para a avaliação das pressões respiratórias máximas.

Descritores: Adolescente; Criança; Obesidade; Ventilação pulmonar.

\begin{abstract}
In this study, it was evaluated changes in pulmonary function in 50 individuals (obese children and adolescents) belonging to two groups: overweight and control, subjected to the test of pulmonary function through the analysis of the following parameters: slow vital capacity (SVL), peak expiratory flow (PEF) and respiratory muscle strength determined by maximum respiratory pressure (Pimax and Pemax). The SVC for the overweight group was $3.0 \pm 0.7$ liters, and the control group of $3.49 \pm 0.9$ liters $(\mathrm{p}=0.0490)$. The PEF for the overweight group of $336 \pm$ 107.4 , and the control group of $386 \pm 55.5(p=0.0404)$. It was concluded that there was a statistically significant change in pulmonary function in obese children and adolescents in relation to slow vital capacity and peak expiratory flow. No difference was observed for the assessment of maximal respiratory pressures.
\end{abstract}

Key words: Adolescent; Child; Obesity; Pulmonary ventilation. 


\section{Introdução}

A obesidade infantil e a do adolescente têm alta prevalência mundial e apresentam caráter multifatorial ${ }^{1,2}$. É reconhecidamente um problema de saúde pública que afeta as sociedades em diversas partes do mundo, caracterizando-se, na atualidade, como uma epidemia ${ }^{3,4}$.

É uma patologia crônica caracterizada pelo acúmulo progressivo de gordura corporal, podendo causar complicações em diversos sistemas do organismo. As doenças que podem estar associadas à obesidade são: hipertensão arterial sistêmica (HAS), diabetes melitos tipo II (DM), dislipidemias, aterosclerose, apnéia obstrutiva do sono e hipoventilação ${ }^{5}$.

Consideráveis repercussões funcionais respiratórias ocorrem em pacientes obesos, tão mais intensas quanto maior o grau da obesidade ${ }^{6,7}$. Postula-se que a obesidade possa exercer efeitos adversos sobre a função ventilatória, mesmo em crianças e adolescentes com obesidade leve ${ }^{8}$.

$\mathrm{O}$ excesso de tecido adiposo promove uma compressão mecânica sobre a caixa torácica, o diafragma, o pulmão e os músculos abdominais, causando redução nas dimensões anatômicas. Além disso, pessoas com elevado peso corporal tendem a ser cronicamente hipoventiladas, têm capacidade aeróbica reduzida, diminuição da complacência pulmonar e maior resistência respiratória levando a uma redução dos volumes e das capacidades pulmonares $3,5,7,8,9,10,11$. Pela ineficácia dos músculos respiratórios na obesidade, a força muscular e a endurance podem ser reduzidas, $\mathrm{o}$ que leva ao aumento do trabalho respiratório, do consumo de $\mathrm{O}_{2}$ e de energia gasta na respiração, fatores que favorecem a respiração superficial ${ }^{5,7}$.

Inúmeros estudos com testes funcionais têm sido desenvolvidos e utilizados para mensuração qualitativa e quantitativa da função pulmonar por meio da avaliação de volumes e fluxos pulmonares ${ }^{12,13}$.

Stirbulov ${ }^{6}$, num estudo de revisão, concluiu que a obesidade causa distúrbios respiratórios; por isso, novas linhas de pesquisas sobre esse tema devem ser incrementadas para deter- minar os mecanismos envolvidos nas repercussões respiratórias da obesidade.

Apesar de vários estudos relatarem as disfunções decorrentes da obesidade sobre o sistema respiratório, faz-se necessário quantificar e descrever as características inerentes a essas situações em nossa região, particularmente na instituição descrita para a realização da pesquisa, uma vez que ela é referência na abordagem e tratamento da obesidade infantil e do adolescente.

A prevenção e/ou detecção precoce de problemas respiratórios em crianças ou adolescentes obesos é fundamental para manter a qualidade de vida desses indivíduos; por isso, no intuito de determinar essas possíveis alterações pulmonares, ressaltamos a relevância desta pesquisa.

\section{Metodologia}

Antes da coleta de dados, os indivíduos e/ou responsáveis receberam esclarecimentos sobre a natureza da pesquisa e autorizaram sua realização assinando o termo de consentimento livre esclarecido formatado de acordo com as normas da resolução 196/96 sobre pesquisa em seres humanos.

Foram avaliados 50 indivíduos pertencentes a dois grupos: sobrepeso, constituído de 25 indivíduos, 14 do sexo masculino, e 11, do feminino, com idade média de 12,7 $\pm 2,7$, variação de 9 a 17 anos; e grupo controle, com 25 indivíduos, 13 do sexo masculino, e 12, do feminino, com idade média de $12,8 \pm 2,8$, variação de 8 a 17 anos.

Os limites de idade foram estipulados conforme o Estatuto da Criança e do Adolescente ${ }^{14}$, que considera criança a pessoa até 12 anos incompletos, e adolescentes, aquelas entre 12 e 18 anos de idade. Já a idade mínima foi determinada de acordo com o grau de dificuldade para a realização dos testes.

O grupo obesidade foi constituído, levando-se em consideração o percentil maior que $85 \%$ referente à tabela de classificação NCHS (National Center for Health Statistic), e a composição do grupo controle foi efetuada utilizan- 
do-se como parâmetro a mesma tabela de classificação com os indivíduos selecionados em uma faixa de $15 \%$ a $85 \% 15$.

O grupo obesidade foi selecionado e avaliado no ambulatório para tratamento da obesidade infantil (TOI) do Hospital Regional do Mato Grosso do Sul (HRMS), localizado em Campo Grande, MS, no período de agosto a setembro de 2008, e o grupo controle, em escola privada de Campo grande, MS, no período de outubro a novembro de 2008.

Inicialmente, as crianças foram pesadas em uma balança da marca Toledo e medidas por meio de um estadiometro fixado à parede; em seguida, foi calculado o IMC com base na equação peso/estatura $^{2}$ (em metro) e, por meio da tabela de classificação da NCHS, calculado o grau de obesidade. Depois, usou-se uma fita antropométrica flexível para medir a circunferência da cintura e do quadril e, então, calculado a razão entre ambas. Aplicou-se também um questionário para detecção de patologias cardiorrespiratórias como meio de exclusão para a composição dos grupos.

Para o teste de função pulmonar, utilizaram-se um ventilômetro da marca Ferraris, modelo Mark 8, de procedência inglesa, um manovacuômetro MV-120 de origem brasileira e um peak flow Meter Assess, de procedência americana.

A ventilometria é usada para obtenção da capacidade vital lenta. Esse teste foi realizado com o paciente na posição sentada com o tronco ereto e os membros inferiores apoiados. Para a execução do exame, foi solicitado que o paciente realizasse uma inspiração máxima seguida de uma expiração forçada lenta por meio do bucal do aparelho, por três vezes, com a seleção do maior resultado.

A manovacuômetria foi realizada para mensuração da Pimáx (pressão inspiratória máxima) e Pemáx (pressão expiratória máxima), com o intuito de verificar a força muscular inspiratória e expiratória, respectivamente. O posicionamento para realização desse teste foi o mesmo da ventilometria. A Pimáx foi mensurada, solicitando-se ao participante uma expiração forçada livre pelo bucal do aparelho, seguida de uma inspiração contra sua resistência. A Pemáx foi mensurada solicitando uma inspiração máxima livre pelo bucal do aparelho, seguida de uma expiração contra a resistência desse instrumento. Também foram feitas três repetições com a aquisição do maior valor.

Por fim, utilizou-se o peak flow ou pico de fluxo expiratório para analisar o nível de obstrução brônquica. Manteve-se o mesmo posicionamento dos testes anteriores. O paciente realizou uma inspiração profunda seguida de uma expiração forçada e rápida. Após três repetições, adquiriu-se o maior valor. Todos os exames foram realizados com obturador nasal.

Tabela 1: Características antropométricas dos sujeitos dos grupos sobrepeso e controle. Dados apresentados em valores médios, desvio-padrão, valor mínimo e móximo. Teste " $\mathrm{t}$ " Student ( $\mathrm{n}=50$ )

\begin{tabular}{|c|c|c|c|}
\hline Varíável & Sobrepeso $(n=25)$ & Controle $(n=25)$ & "p" \\
\hline \multirow[t]{2}{*}{ Idade (anos) } & $12,7 \pm 2,7$ & $12,8 \pm 2,8$ & \multirow{2}{*}{$0,9185 \mathrm{~ns}$} \\
\hline & $9-17$ & $8-17$ & \\
\hline \multirow[t]{2}{*}{ Peso $(\mathrm{Kg})$} & $86,6 \pm 31,0$ & $46,6 \pm 11,4$ & \multirow{2}{*}{$<0,0001^{* * *}$} \\
\hline & $47,0-164,5$ & $27,7-67,6$ & \\
\hline \multirow[t]{2}{*}{ Altura (metros) } & $1,58 \pm 0,1$ & $1,56 \pm 0,1$ & \multirow{2}{*}{$0,6038 \mathrm{~ns}$} \\
\hline & $1,36-1,79$ & $1,30-1,77$ & \\
\hline \multirow[t]{2}{*}{ IMC (Kg/cm2) } & $33,7 \pm 8,2$ & $18,7 \pm 2,1$ & \multirow{2}{*}{$<0,0001 * * *$} \\
\hline & $22,4-56,3$ & $15,6-23,4$ & \\
\hline \multirow[t]{2}{*}{ Razão cintura-quadril (cm) } & $0,86 \pm 0,1$ & $0,73 \pm 0,1$ & \multirow{2}{*}{$<0,0001 * * *$} \\
\hline & $0,73-1,02$ & $0,62-0,87$ & \\
\hline
\end{tabular}

ns (não siginificativo); ${ }^{* * *}$ (estatisticamente muito significativo) 
Tabela 2: Avaliação da função pulmonar entre os sujeitos dos grupos sobrepeso e controle. Dados apresentados em valores médios, desvio-padrão, valor mínimo e móximo. Teste " $\mathrm{t}$ " Student $(\mathrm{n}=50)$

Variável

Capacidade vital lenta (litros)

Pico fluxo expiratório (L/min)

Pressão inspiratória máxima $(\mathrm{cmH2O})$

Pressão expiratória máxima (cmH2O)

\section{Sobrepeso \\ $\mathbf{n}=25$}

$3,0 \pm 0,7$

$1,7-4,7$

$336 \pm 107,4$

$200-690$

$85 \pm 20,2$

$52-120$

$85 \pm 16,9$

$56-116$

\section{Controle \\ $\mathbf{n}=\mathbf{2 5}$}

$3,49 \pm 0,9$

$2,08-5,07$

$386,9 \pm 55,5$

$254-454$

$81,0 \pm 19,2$

$60-120$

$90,7 \pm 12,4$

$70-120$ "p"

$0,0490 *$

$0,0404^{*}$

$0,4255 \mathrm{~ns}$

$0,1486 \mathrm{~ns}$

ns (não siginificativo); * (estatisticamente muito significativo)

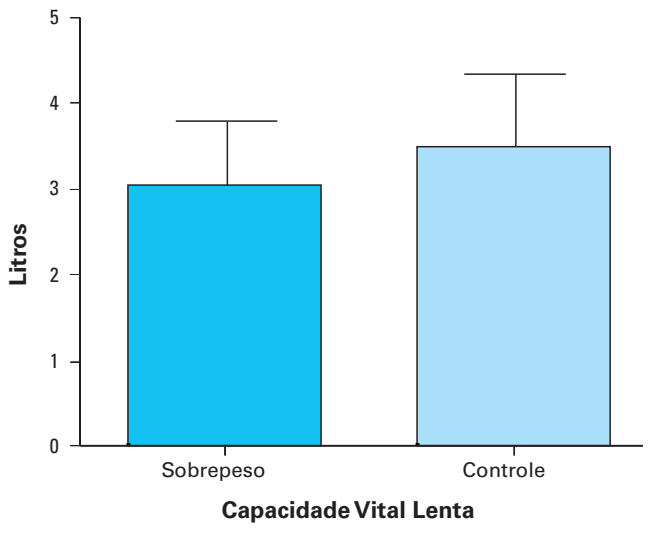

Figura 1: Valores médios e desvio-padrão da capacidade vital lenta dos grupos sobrepeso e controle $(n=50)$

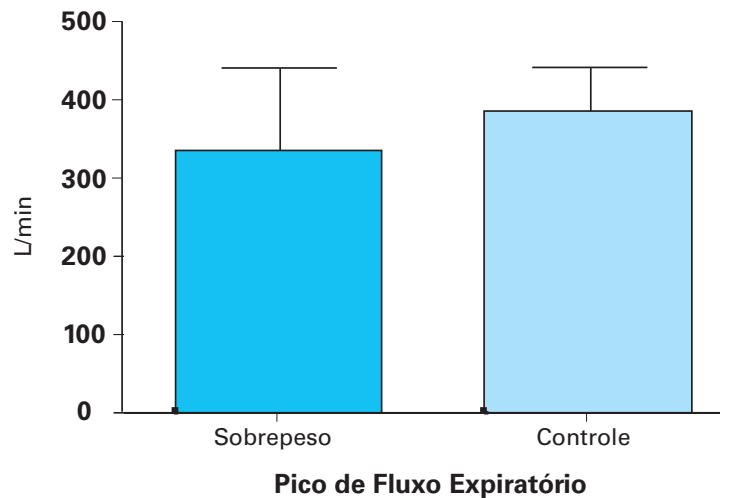

Figura 2: pico de fluxo expiratório dos grupos sobrepeso e controle. Valores expressos em média e desvio-padrão $(n=50)$

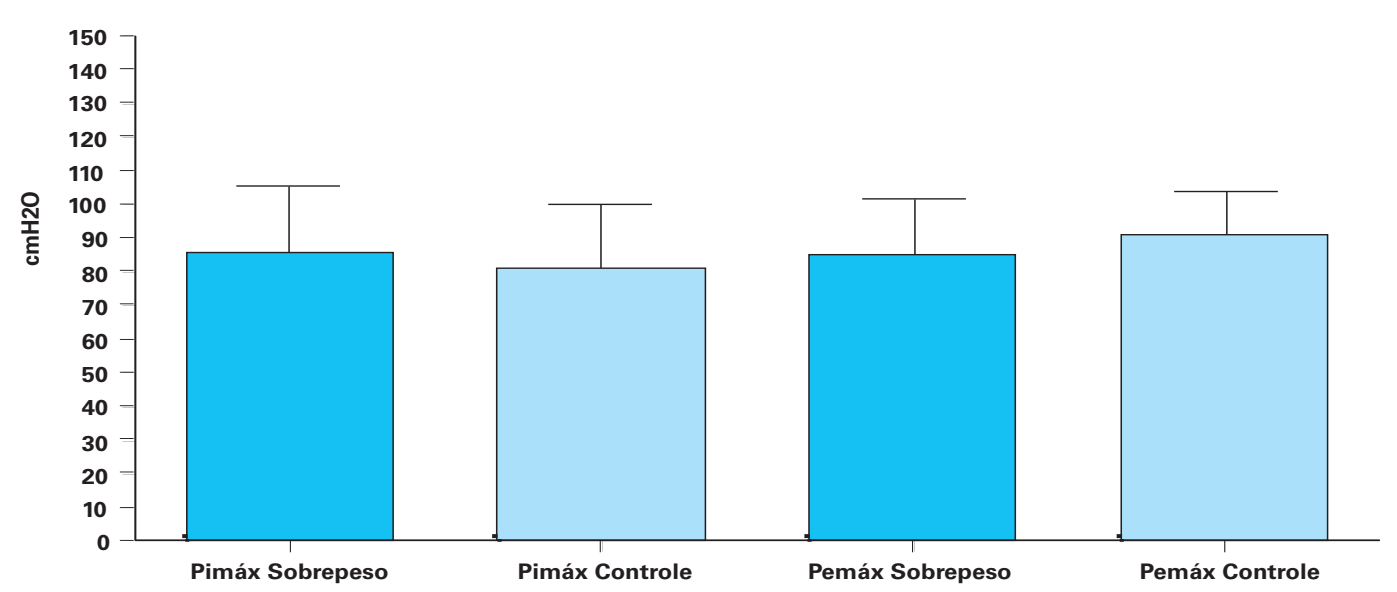

Figura 3: Pressões respiratórias máximas dos grupos sobrepeso e controle. Valores expressos em média e desvio-padrão $(n=50)$ 
Para análise estatística, os valores da ventilometria, da manovacuometria e do pico de fluxo expiratório foram comparados entre os grupos por meio do teste " $\mathrm{t}$ " Student para amostras não- pareadas, com nível de decisão estabelecido em $\mathrm{p}<0,05$.

\section{Resultados}

Os indivíduos do grupo controle foram selecionados e comparados com os indivíduos do grupo sobrepeso pelas variáveis idade, altura e sexo.

As medidas antropométricas dos voluntários do grupo sobrepeso e controle estão apresentadas na Tabela 1. Os valores referentes à função pulmonar comparada entre os dois grupos estão expostos na Tabela 2.

\section{Discussão}

A proposta deste estudo foi avaliar alterações na função pulmonar em crianças e adolescentes obesos comparada as dos indivíduos com peso corporal dentro dos limites normais por meio das medidas da capacidade vital lenta, pico de fluxo expiratório e força muscular respiratória.

A obesidade introduz importantes alterações na mecânica respiratória. Na presença de obesidade excessiva, ocorre redução da capacidade pulmonar total e da capacidade vital forçada, constituindo um quadro restritivo ${ }^{72,16}$. A eventual redução dos fluxos aéreos é consequência da redução da capacidade vital forçada, mas nem sempre isso ocorre, sendo difícil prever o efeito da obesidade sobre a função pulmonar ${ }^{12}$.

Os dados observados neste estudo demonstraram uma capacidade vital lenta com redução de $14,3 \%$ nas crianças e adolescentes obesos em relação aos não-obesos. Oliveira et $\mathrm{al}^{12}$, descreveram, por meio da análise dos volumes pulmonares que os indivíduos obesos apresentavam comportamento restritivo, com redução desses volumes que, em casos de obesidade acentuada, pode alcançar 20 a 30\% da capacidade pulmonar total e da vital. Tal evidência pode ser explicada tomando-se como base a hipótese de que o excesso de tecido adiposo interfere na movimentação do diafragma e da excursão da parede torácica ${ }^{17}$.

Também se verificou alteração significante do pico de fluxo expiratório que se apresentou diminuído nas crianças e adolescentes obesos em relação aos indivíduos com peso corporal normal. Pereira ${ }^{17}$ descreve que a capacidade vital forçada reduzida leva à diminuição da $\mathrm{VEF}_{1}$ (volume expiratório no primeiro segundo) em quadros não-obstrutivos como a dos obesos, levando à diminuição do pico de fluxo expiratório, em decorrência da redução do volume expirado.

A Pimáx e a Pemáx têm sido utilizadas, com eficiência, para mensuração da força muscular respiratória. A Pemáx é uma medida que indica a força dos músculos abdominais e intercostais enquanto que a Pimáx indica a força do músculo diafragma9.

Paisani et al. ${ }^{7}$ realizaram um estudo com 30 indivíduos adultos candidatos à gastroplastia pela técnica descrita como Capella e observaram uma grande variabilidade entre os valores da Pimáx e da Pemáx, com resultados dentro da normalidade para não-obesos ou até com valores acima do esperado. Resultados semelhantes ocorreram neste trabalho em que não houve diferenças estatísticas entre os grupos avaliados. Essa pequena repercussão nos valores da Pimáx e da Pemáx pode ser explicada pelo fato de os obesos apresentarem maior sobrecarga inspiratória, promovendo, dessa forma, um efeito de treinamento sob a musculatura respiratória.

A localização do depósito de gordura influencia as alterações da função ventilatória, sendo essas anormalidades mais comuns na obesidade central, em que o acúmulo de tecido adiposo se localiza na região da cintura e, provavelmente, exerce um efeito mecânico direto na caixa torácica e no diafragma, restringindo a expansibilidade pulmonar e causando redução dos volumes pulmonares ${ }^{3,7}$. Isso demonstra a importância da avaliação da razão cintura-quadril que, além de determinar a obesidade central, está envolvida 
com os riscos das doenças cardiovasculares e o aumento dos sintomas respiratórios.

Vale ressaltar que, na literatura atual, são escassos os estudos referentes aos efeitos da obesidade na criança e no adolescente, tornando-se necessária a realização de novas pesquisas que determinem o real efeito do excesso de tecido adiposo na função ventilatória desses indivíduos.

\section{Conclusão}

Houve alteração estatisticamente significativa na função pulmonar das crianças e dos adolescentes obesos em relação à capacidade vital lenta e ao pico de fluxo expiratório, evidenciando, na amostra estudada, alterações nas propriedades ventilométricas pulmonares causadas pela obesidade. Não foi observada diferença para avaliação das pressões respiratórias máximas.

\section{Referências}

1. Oliveira CL, Fisberg M. Obesidade na infância e adolescência - uma verdadeira epidemia. Arq Bras Endocrinol Metab. 2003;47(2):107-08.

2. Oliveira AMA, Cerqueira EMM, Souza JS, Oliveira AC. Sobrepeso e obesidade infantil: influência de fatores biológicos e ambientais em Feira de Santana, BA. Arq Bras Endocrinol Metab. 2003;47(2):144-50.

3. Rigatto AM, Alves SCC, Gonçalves CB, Firmo JF, Provin LM. Performance Ventilatória na Obesidade. Saúde Ver. 2005;7(17):57-62.

4. Pitanga FJG, Lessa I. Razão cintura-estatura como discriminador do risco coronariano de adultos. Ver Assoc Med Bras. 2006;52(3):157-61.

5. Lewandoski LT. Aplicação de um protocolo fisioterapêutico em pacientes obesos com indicação à cirurgia bariátrica. [Monografia]. Curso de Fisioterapia Unoeste. Cascavel, Paraná. 2005.

6. Stirbulov, R. Repercussões respiratórias da obesidade. J Bras Pneumol. 2007;33(1):7-8.
7. Paisani DM., Chiavegato LD. Faresin, S. M. Volumes, capacidades pulmonares e força muscular respiratória no pós-operatório de gastroplastia. J Bras Pneumol. 2005;31(2):125-32.

8. Boran P, Tokuc G, Pisgin B, Oktem S, Yegin Z, Bostan O. Impact of obesity on ventilatory function. J Pediatr. 2007;83(2):171-76.

9. Costa D, Sampaio LMM, Lorenzo VAP, Jamami M, Damaso AR. Avaliação da força muscular respiratória e amplitudes torácicas e abdominais após a RFR em indivíduos obesos. Rev Latino-am Enfermagem. 2003;11(2):156-60.

10. Faintuch J, Souza SAF, Valezi AC, Sant'Anna AF, Rodrigues JJG. Pulmonary function and aerobic capacity in asymptomatic bariatric candidates with very severe morbid obesity. Rev Hosp Clín Fac Med São Paulo. 2004;59(4):181-86.

11. Rasslan Z, Saad Junior R, Stirbulov R, Fabbri RMA, Lima CAC. Avaliação da função pulmonar na obesidade graus I e II. J Bras Pneumol. 2004;30(6):508-14.

12. Oliveira FB, Aguiar LGK, Bouskela E, Jansen JM, Melo PL. Análise do efeito da obesidade sobre as propriedades resistivas e elásticas do sistema respiratório por oscilações forçadas. Pulmão RJ. 2006;15(4):219-23.

13. Terra Filho J. Avaliação laboratorial da função pulmonar. In: Simpósio de doenças pulmonares; 1998; Ribeirão Preto, BR. Riberão Preto: Faculdade de Medicina da Universidade de São Paulo, 1998. p.191-207.

14. Estatuto da criança e do adolescente. [acesso em: 20 out. 2008]. Disponível em: http://www.planalto.gov. br/ccivil/LEIS/L8069.htm

15. Shils ME, Olson JA, Shike M, Ross AC. Tratado de nutriação moderna na saúde e na doença. $9^{a}$ ed. Barueri: Manole; 2003.

16. Silva AMO, Boin IFS, Pareja JC, Magna LA. Análise da função respiratória em pacientes obesos submetidos à operação Fobi-Capella. Ver Col Bras Cir. 2007; 34(5):314-20.

17. Sociedade Brasileira de Pneumologia e Tisiologia (SBPT). I Consenso brasileiro sobre espirometria. J Pneumol. 1996;22:105-64. 